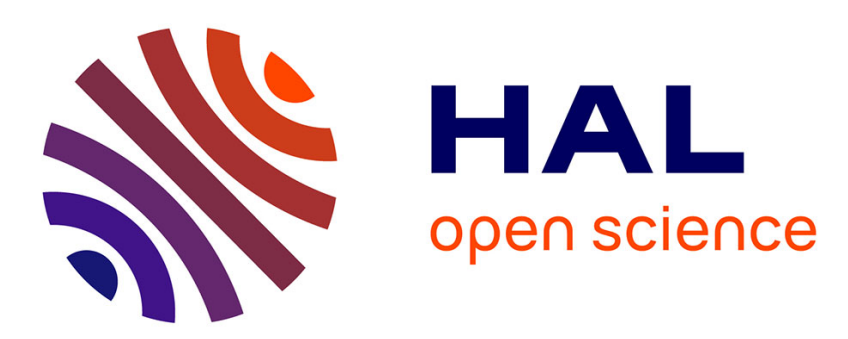

\title{
Global mode analysis of axisymmetric bluff-body wakes: Stabilization by base bleed
}

E. Sanmiguel-Rojas, A. Sevilla, C. Martínez-Bazán, Jean-Marc Chomaz

\section{To cite this version:}

E. Sanmiguel-Rojas, A. Sevilla, C. Martínez-Bazán, Jean-Marc Chomaz. Global mode analysis of axisymmetric bluff-body wakes: Stabilization by base bleed. Physics of Fluids, 2009, 21, pp.114102. 10.1063/1.3259357 . hal-01002591

HAL Id: hal-01002591

https://hal-polytechnique.archives-ouvertes.fr/hal-01002591

Submitted on 7 Jul 2014

HAL is a multi-disciplinary open access archive for the deposit and dissemination of scientific research documents, whether they are published or not. The documents may come from teaching and research institutions in France or abroad, or from public or private research centers.
L'archive ouverte pluridisciplinaire HAL, est destinée au dépôt et à la diffusion de documents scientifiques de niveau recherche, publiés ou non, émanant des établissements d'enseignement et de recherche français ou étrangers, des laboratoires publics ou privés. 


\section{AIP | Physics of Fluids}

\section{Global mode analysis of axisymmetric bluff-body wakes: Stabilization by base bleed}

E. Sanmiguel-Rojas, A. Sevilla, C. Martínez-Bazán, and J.-M. Chomaz

Citation: Physics of Fluids (1994-present) 21, 114102 (2009); doi: 10.1063/1.3259357

View online: http://dx.doi.org/10.1063/1.3259357

View Table of Contents: http://scitation.aip.org/content/aip/journal/pof2/21/11 ?ver=pdfcov

Published by the AIP Publishing

\section{Articles you may be interested in}

Effect of base cavities on the stability of the wake behind slender blunt-based axisymmetric bodies

Phys. Fluids 23, 114103 (2011); 10.1063/1.3658774

Bifurcations and symmetry breaking in the wake of axisymmetric bodies

Phys. Fluids 20, 051702 (2008); 10.1063/1.2909609

A note on the stabilization of bluff-body wakes by low density base bleed

Phys. Fluids 18, 098102 (2006); 10.1063/1.2355655

Vortex shedding in high Reynolds number axisymmetric bluff-body wakes: Local linear instability and global bleed control

Phys. Fluids 16, 3460 (2004); 10.1063/1.1773071

A coupled Landau model describing the Strouhal-Reynolds number profile of a three-dimensional circular cylinder wake

Phys. Fluids 15, L68 (2003); 10.1063/1.1597471

\section{A|P| $\left.\right|_{\text {Applied Physics }} ^{\text {Journal of }}$}

Journal of Applied Physics is pleased to announce André Anders as its new Editor-in-Chief 


\title{
Global mode analysis of axisymmetric bluff-body wakes: Stabilization by base bleed
}

\author{
E. Sanmiguel-Rojas, ${ }^{1}$ A. Sevilla, ${ }^{1}$ C. Martínez-Bazán, ${ }^{1}$ and J.-M. Chomaz ${ }^{2}$ \\ ${ }^{1}$ Departamento de Ingeniería Mecánica y Minera, Área de Mecánica de Fluidos, Universidad de Jaén, \\ Campus de las Lagunillas, 23071 Jaén, Spain \\ ${ }^{2}$ Laboratoire d'Hydrodynamique (LadHyx), CNRS-Ecole Polytechnique, 91128 Palaiseau, France
}

(Received 17 February 2009; accepted 7 October 2009; published online 6 November 2009)

\begin{abstract}
The flow around a slender body with a blunt trailing edge is unstable in most situations of interest. Usually the flow instabilities are generated within the wake behind the bluff body, inducing fluctuating forces and introducing the possibility of resonance mechanisms with modes of the structure. Base bleed is a simple and well-known means of stabilizing the wake. In the present research, we investigate the global instability properties of the laminar-incompressible flow that develops behind a cylinder with sharp edges and axis aligned with the free stream using a spectral domain decomposition method. In particular, we describe the flow instability characteristics as a function of the Reynolds number, $\operatorname{Re}=\rho W_{\infty} D / \mu$, and the bleed coefficient, defined as the bleed-to-free-stream velocity ratio, $C_{b}=W_{b} / W_{\infty}$, where $D$ is the diameter of the body and $\rho$ and $\mu$ the density and viscosity of the free stream, respectively. For a truncated cylinder of aspect ratio $L / D=5$, where $L$ is the length of the body, our calculations reveal the presence of a first steady bifurcation in the wake at $\operatorname{Re} \simeq 391$, as well as a second oscillatory one at $\operatorname{Re} \simeq 715$ with an associated Strouhal number $\mathrm{St} \simeq 0.0905$ for the most unstable azimuthal mode $|m|=1$. In addition, we report the existence of two critical values of the bleed coefficient $C_{b 1}^{*}(\operatorname{Re},|m|)$ and $C_{b 2}^{*}(\operatorname{Re},|m|)<C_{b 1}^{*}$, which vary with the aspect ratio of the body, needed to stabilize both the first and second bifurcations in the range of Reynolds numbers under study, $0 \leq \operatorname{Re} \leq 2200$. Finally, the numerical results for the oscillatory mode obtained for a bulletlike body of aspect ratio $L / D=2$ without base bleed are compared with experiments performed in a wind tunnel using hot-wire anemometry, showing the limitations of using an axisymmetric basic flow at Reynolds numbers higher than the critical one corresponding to the first steady bifurcation in the global stability analysis. (C) 2009 American Institute of Physics. [doi:10.1063/1.3259357]
\end{abstract}

\section{INTRODUCTION}

The study of wakes behind bluff bodies is still one of the most active research fields in fluid mechanics after many decades of intense investigation. The widespread importance of the vortex shedding phenomenon in nature and technological applications, as well as its surprisingly rich and geometry-dependent dynamics, justify the intense research performed over the years to understand its basic features, as well as to assess efficient mechanisms for its control. From a fundamental point of view, it is now clear that vortex shedding behind two-dimensional and axisymmetric bluff bodies is the result of a global instability associated with the structure of the near wake, whose main feature is the presence of a recirculating bubble. In a pioneering study, Zebib ${ }^{1}$ investigated numerically the global linear stability of the steady symmetric wake behind a circular cylinder and determined the critical value of the Reynolds number, as well as the Strouhal number associated with the most unstable global eigenmode for which both the cross-stream and the streamwise directions are eigendirections. At the same time, Monkewitz and Nguyen ${ }^{2}$ demonstrated that a region of absolute instability is present in the near wake of twodimensional bluff bodies, which was associated with the occurrence of vortex shedding. Absolute instability is a local concept strictly valid when the flow is parallel and the perturbations may be expanded in traveling waves in the streamwise direction. Thus, it refers to the local behavior of a wave packet and it is only valid when basic flow variations take place at scales much larger than the instability scales. In contrast, global stability does not make such approximation and considers the streamwise direction as an eigendirection. More generally, recent studies have established a link between the presence of regions of local absolute instability in spatially developing flows and the onset of unstable global modes, see Chomaz. ${ }^{3}$ Moreover, many techniques have been developed in order to control the vortex shedding phenomenon, see Choi et al. ${ }^{4}$ and references therein.

In the present work we consider the global linear stability of the wake behind an axisymmetric bluff body with a blunt trailing edge of axis aligned with the free stream, as well as its possible stabilization by means of base bleed, as already studied by Sevilla and Martínez-Bazán ${ }^{5}$ from the local point of view. Both the basic axisymmetric, steady flow, as well as its global linear stability, is numerically calculated by means of a spectral domain decomposition method. The paper is structured as follows. In Sec. II we describe the numerical methods developed to calculate the basic flow and to perform the stability analysis. Section III summarizes the results obtained from the global stability analyses for a cyl- 


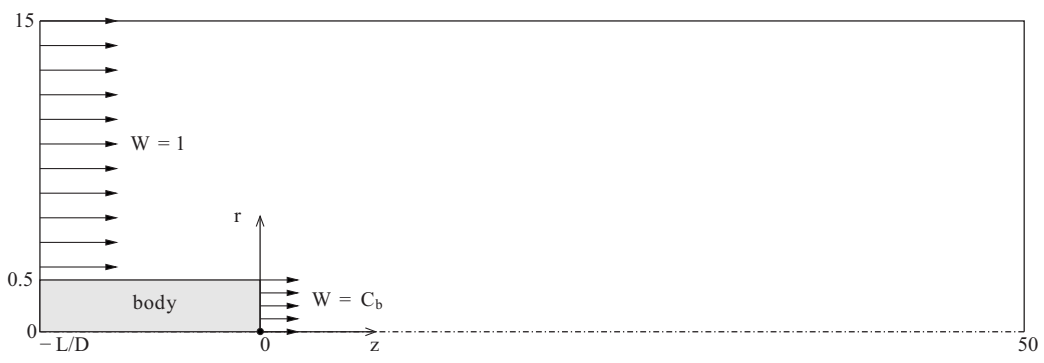

FIG. 1. Nondimensional computational domain.

inder with sharp edges and axis aligned with the flow and describes the effects of the Reynolds number Re, the aspect ratio $L / D$, and the bleed coefficient $C_{b}$ on the stability properties of the flow. In Sec. IV we present the experimental results obtained in a low speed wind tunnel for a bulletlike body of aspect ratio $L / D=2$ without base bleed and compare them with presently computed global stability properties of the axisymmetric basic flow. Finally, Sec. V is devoted to the conclusions, including a discussion of the implications of our linear results to the nonlinear dynamics of the wake, as well as a comparison to recent related works.

\section{NUMERICAL METHOD}

\section{A. Basic flow calculation}

The flow configuration under study here, sketched in Fig. 1, consists of a uniform fluid stream of density $\rho$ and viscosity $\mu$, flowing with a free stream velocity $W_{\infty}$ around a cylinder of diameter $D$ and length $L$, into a coaxial cylindrical surface of radius $15 D$ and length $L+50 D$, which represents the fluid field far away from the body. The equations were made dimensionless using $D, W_{\infty}, D / W_{\infty}$, and $\rho W_{\infty}^{2}$ as length, velocity, time, and pressure scales, respectively, in cylindrical-polar coordinates $(r, \theta, z)$ with velocity field given by $\mathbf{v}=(u, v, w)$. To tackle the global stability analysis, the flow was decomposed into a basic steady-state field with zero swirl and a perturbed field as

$$
(\mathbf{v}, p)=\left(\mathbf{V}+\mathbf{v}^{\prime}, P+p^{\prime}\right),
$$

where $\mathbf{V}=[U(r, z), 0, W(r, z)]$ and $P(r, z)$ are the velocity and the pressure fields of the basic axisymmetric steady flow, and $\mathbf{v}^{\prime}=\left(u^{\prime}, v^{\prime}, w^{\prime}\right)$ and $p^{\prime}$ represent the small amplitude, unsteady velocity, and pressure perturbation fields, respectively. The steady-state solution was calculated substituting Eq. (1) into the incompressible Navier-Stokes equations, and enforcing to zero the steady and axisymmetric terms to yield

$$
\nabla \cdot \mathbf{V}=0,
$$

$$
\mathbf{V} \cdot \nabla \mathbf{V}+\nabla P-\frac{1}{\operatorname{Re}} \nabla^{2} \mathbf{V}=0
$$

where $\operatorname{Re}=\rho W_{\infty} D / \mu$ is the Reynolds number. The boundary conditions to solve Eqs. (2) and (3) are given by (see Fig. 1)

$$
\begin{aligned}
& 0<r<0.5, \quad z=0: \quad U=0, \quad W=C_{b}, \\
& r=0.5, \quad-L / D \leq z \leq 0: \quad U=W=0, \\
& 0.5<r \leq 15, \quad z=-L / D: \quad U=0, \quad W=1,
\end{aligned}
$$

$$
\begin{aligned}
& r=15, \quad-L / D<z<50: \quad U=\partial W / \partial r=0, \\
& 0<r \leq 15, \quad z=50: \quad \boldsymbol{\tau}_{i j} \cdot \mathbf{n}=0, \\
& r=0, \quad 0 \leq z \leq 50: \quad U=\partial W / \partial r=0,
\end{aligned}
$$

where the base bleed velocity profile was assumed to be uniform with $C_{b}=W_{b} / W_{\infty}$ being the bleed-to-free-stream velocity ratio, commonly called bleed coefficient, $\boldsymbol{\tau}_{i j}$ is the stress tensor, and $\mathbf{n}$ is the normal surface vector. The pressure values in the boundary conditions (4)-(9) are implicitly obtained through the continuity equation (2) (see Deville et al. ${ }^{6}$ for a detailed description). Here, Eq. (8) corresponds to the stress-free condition applied at the outflow surface of the computational domain.

Notice that the control parameters of the problem at hand are the Reynolds number, for which we considered the range $0 \leq \operatorname{Re} \leq 2200$, the bleed coefficient, which was varied in the range $0 \leq C_{b} \leq 0.1$, and the aspect ratio of the body $L / D$. Since the purpose of this work was to study in detail the effects of the Reynolds number and the bleed coefficient on the stability of the wake for a given geometry, the aspect ratio was fixed at a value $L / D=5$ in all the calculations performed with base bleed $C_{b}>0$, which are presented in Sec. III B. However, in the particular case without base bleed, $C_{b}=0$, considered in Sec. III A, we have also studied the influence of the geometry by varying the aspect ratio in the range $2 \leq L / D \leq 10$.

The basic flow was computed using the NewtonRaphson method, ${ }^{7}$ and the Chebyshev collocationdecomposition method was used to discretize and solve both the basic flow and the stability equations. ${ }^{8,9}$ Basically, the decomposition method consists of dividing the computational domain into a block-structured grid with fictitious overlapping interfaces. In our calculations, the domain was divided into 314 subdomains whose size increased with the distance from the body since the physical gradients are smaller than in the near field, and, thus the resolution can be decreased [see Fig. 2(a)]. Moreover, the functions were approximated by $n \times n$ tensor-product Lagrangian polynomials in the local computational space $[-1,1] \times[-1,1]$, and fitted using $(n+1) \times(n+1)$ nodes distributed according to the Gauss-Lobatto-Chebyshev points. An example of the basic flow structure can be observed in Figs. 3(a) and 3(b), which display the streamlines obtained for an aspect ratio $L / D=5$ at $\operatorname{Re}=1000$ and two different values of the bleed coefficient, namely, $C_{b}=0$ and $C_{b}=0.090$, respectively. Notice that the main effect of base bleed is, on the one hand, to push the 

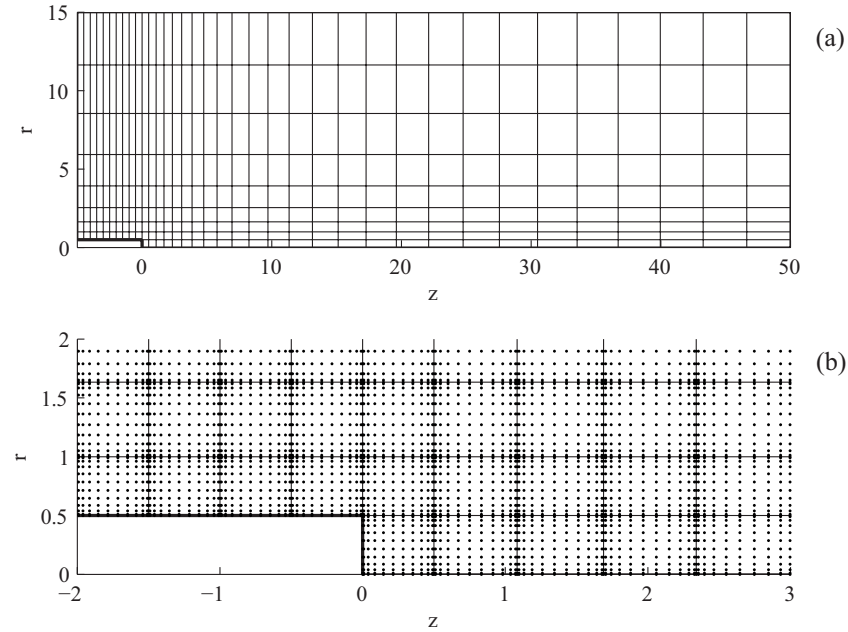

FIG. 2. (a) Domain decomposition into 314 subdomains. The thin solid lines correspond to the subdomain boundaries. (b) Grid detail close to the body for a polynomial order of $n=11$.

recirculation bubble downstream from the body base, and, on the other hand, to decrease the velocity defect in the wake.

\section{B. Stability analysis}

Once the steady basic flow was determined, the linearized equations that describe the perturbation field were obtained by substituting Eq. (1) into the incompressible Navier-Stokes equations to yield

$$
\begin{aligned}
& \nabla \cdot \mathbf{v}^{\prime}=0, \\
& \mathbf{V} \cdot \nabla \mathbf{v}^{\prime}+\mathbf{v}^{\prime} \cdot \nabla \mathbf{V}+\nabla p^{\prime}-\frac{1}{\operatorname{Re}} \nabla^{2} \mathbf{v}^{\prime}=-\frac{\partial \mathbf{v}^{\prime}}{\partial t} .
\end{aligned}
$$

Equations (10) and (11) can formally be expressed as
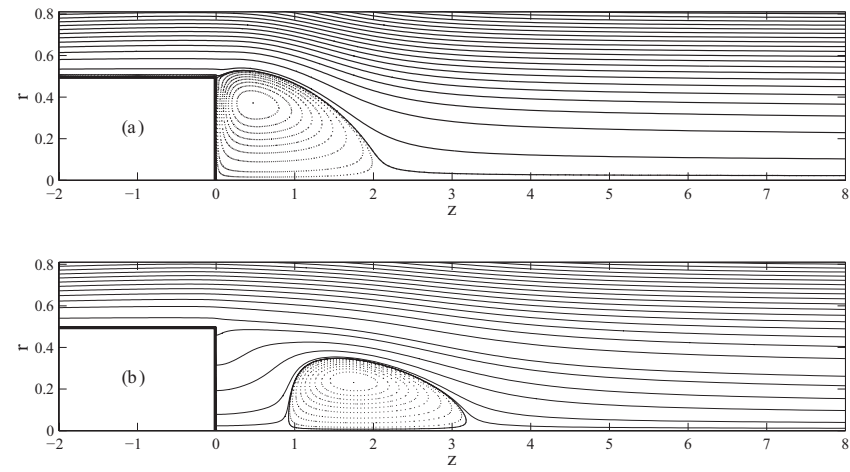

FIG. 3. Streamlines of the basic flow for $L / D=5$ and $\mathrm{Re}=1000$ (a) without base bleed $\left(C_{b}=0\right)$ and (b) with base bleed $\left(C_{b}=0.090\right)$.

$$
\mathcal{A} \mathbf{q}=\partial / \partial t \mathcal{B q}
$$

where $\mathbf{q}=\left[\mathbf{v}^{\prime}, p^{\prime}\right]^{T}$ represents the total disturbance field, and $\mathcal{A}$ and $\mathcal{B}$ are linear matrix operators. Assuming both time and azimuthal exponential dependencies, solutions for $\mathbf{q}$ of the form

$$
\mathbf{q}=[\hat{\mathbf{v}}(r, z), \hat{p}(r, z)]^{T} e^{\sigma t+i m \theta}=\hat{\mathbf{q}}(r, z) e^{\sigma t+i m \theta}
$$

can be sought, where the variables with a hat, $\hat{\mathbf{v}}, \hat{p}$, and $\hat{\mathbf{q}}$, represent the eigenfunctions, $m$ is the azimuthal mode, and $\sigma=\sigma_{r}+i \sigma_{i}$ with $\sigma_{r}$ the growth rate of the global mode and $\sigma_{i}$ its angular frequency. Moreover, with the ansatz of Eq. (13) the initial value problem defined in Eq. (12) can be rewritten as a generalized eigenvalue problem given by

$$
\mathcal{A}_{m} \hat{\mathbf{q}}=\sigma \mathcal{B}_{m} \hat{\mathbf{q}}
$$

with

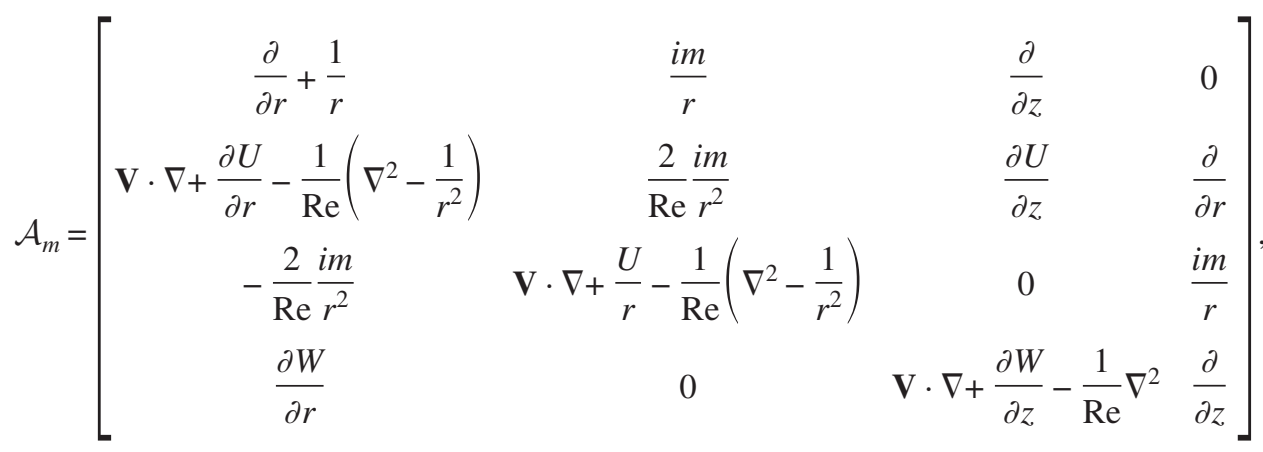

$\mathcal{B}_{m}=-\left[\begin{array}{llll}0 & 0 & 0 & 0 \\ 0 & I & 0 & 0 \\ 0 & 0 & I & 0 \\ 0 & 0 & 0 & I\end{array}\right]$.

number of grid points. After discretizing the eigenvalue problem of Eq. (14), a grid convergence study was performed to select the optimal size of the domain and value of the polynomial order $n$ in terms of accuracy and computational cost. The convergence test was performed for $L / D$ $=5, \operatorname{Re}=1000, C_{b}=0$, and $|m|=1$, a case that presents two different bifurcations, as described in detail in Sec. III. Ini-
In Eqs. (15) and (16), $I$ and 0 represent, respectively, the identity and null matrices of order $n_{g} \times n_{g}$, where $n_{g}$ is the 
TABLE I. Grid convergence study for the two unstable eigenvalues found for $L / D=5, \operatorname{Re}=1000, C_{b}=0$, and $|m|=1$ using five different mesh sizes. The computational domain extended from $z=-5$ to $z=50$ and from $r$ $=0$ to $r=15$.

\begin{tabular}{cccccc}
\hline \hline Mesh & $n$ & Subdomains & Spectral nodes & $\sigma_{1}$ & $\sigma_{2}$ \\
\hline 1 & 8 & 314 & 20457 & 0.21723 & $0.09123+0.55235 i$ \\
2 & 9 & 314 & 25840 & 0.20312 & $0.08156+0.58433 i$ \\
3 & 10 & 314 & 31851 & 0.19328 & $0.07594+0.56284 i$ \\
$\mathbf{4}$ & $\mathbf{1 1}$ & $\mathbf{3 1 4}$ & $\mathbf{3 8 4 9 0}$ & $\mathbf{0 . 1 8 0 9 0}$ & $\mathbf{0 . 0 6 9} \mathbf{8 1 + 0 . 5 5 9 0 5 i}$ \\
5 & 12 & 314 & 45757 & 0.18071 & $0.06927+0.55302 i$ \\
\hline \hline
\end{tabular}

tially, for a domain that extended from $z=-5$ to $z=50$ and from $r=0$ to $r=15$, we used five meshes with the same number of subdomains, 314, and different values of $n$. Table I shows the convergence of the growth rate of the most unstable mode number corresponding to a stationary bifurcation $\sigma_{1}$, as well as the growth rate and the angular frequency of the second unstable mode number corresponding to the oscillatory bifurcation $\sigma_{2}$. It can be observed in this table that incrementing the polynomial order from $n=11$ to $n=12$ introduces differences of $\mathrm{O}\left(10^{-4}\right)$ in $\sigma_{1}$ and of $\mathrm{O}\left(10^{-3}\right)$ in $\sigma_{2}$, but the computational time of the simulations increases by a factor of 3 . Therefore, $n=11$ was selected to perform the calculations discussed below. The effect of changing the size of the computational domain was also investigated by keeping constant the radius of the domain at $r=15$, and varying its length from $z=40$ to $z=100$, obtaining that the eigenvalue spectrum became nearly independent of the length of the domain for $z \approx 50$. Notice that the relative differences among the eigenvalues obtained with a domain of length $z=50$ (mesh 2 in Table II) and those obtained doubling the length to $z=100$ (mesh 4 in Table II) are smaller than 1\%. A similar study was performed fixing the length of the computational domain at $z=50$ and varying its radius from $r=10$ to $r=20$. In this case, the spectra did not show any significant changes when the radius of the domain was lager than 15 . Thus, $r$ $=15$ and $z=50$ were, respectively, selected as radial and longitudinal dimensions of the computational domain. The results obtained with the different computational domains are summarized in Table II, indicating that the values of $\sigma_{1}$ and $\sigma_{2}$ are converged for $r \geq 15$ and $z \geq 50$. Finally, to make sure that the code conveniently resolved the flow at the inlet $(r$ $=0.5, z=-5)$ and the rear corner $(r=0.5, z=0)$, we increased the number of grid points in the vicinity of the singular regions [see Fig. 2(b)].

Note that mesh 4 in Table I consists of 38490 nodes, which represents a problem too large to be solved with a standard QZ-algorithm. Therefore, the Arnoldi method based on the Krylov subspace was used to reduce the size of the matrix and to find a few eigenvalues. ${ }^{10,11}$ To look for unstable modes in a wide range of the complex plane with a relatively small size of the Krylov subspace, it was necessary to apply the shift and invert strategy. This method allowed us to obtain the eigenvalue spectrum in the neighborhood of a shift parameter $\beta$ and, thus, to transform the generalized eigenvalue problem of Eq. (14) into the following standard one:

$$
\mathcal{M} \hat{\mathbf{q}}=\lambda \hat{\mathbf{q}}, \quad \text { with }\left\{\begin{array}{l}
\mathcal{M}=\left(\mathcal{A}_{m}-\beta \mathcal{B}_{m}\right)^{-1} \mathcal{B}_{m}, \\
\lambda=1 /(\sigma-\beta),
\end{array}\right.
$$

with the boundary conditions

$$
\begin{aligned}
& 0<r<0.5, \quad z=0: \quad \hat{u}=\hat{v}=\hat{w}=0, \\
& r=0.5, \quad-L / D \leq z \leq 0: \quad \hat{u}=\hat{v}=\hat{w}=0, \\
& 0.5<r \leq 15, \quad z=-L / D: \quad \hat{u}=\hat{v}=\hat{w}=0, \\
& r=15, \quad-L / D<z<50: \quad \hat{u}=\hat{v}=\partial \hat{w} / \partial r=0, \\
& 0<r \leq 15, \quad z=50: \quad \hat{\boldsymbol{\tau}}_{i j} \cdot \mathbf{n}=0,
\end{aligned}
$$

TABLE II. Grid convergence study for the two unstable eigenvalues found for $L / D=5, \operatorname{Re}=1000, C_{b}=0$, and $|m|=1$ using different computational domains and $n=11$.

\begin{tabular}{ccrccc}
\hline \hline Mesh & $r$ & $z$ & Subdomains & $\sigma_{1}$ & $\sigma_{2}$ \\
\hline 1 & 15 & 40 & 287 & 0.19032 & $0.07123+0.54310 i$ \\
$\mathbf{2}$ & $\mathbf{1 5}$ & $\mathbf{5 0}$ & $\mathbf{3 1 4}$ & $\mathbf{0 . 1 8 0} \mathbf{9 0}$ & $\mathbf{0 . 0 6 9} \mathbf{8 1 + 0 . 5 5 9 ~ 0 5 i}$ \\
3 & 15 & 60 & 341 & 0.18081 & $0.06875+0.56274 i$ \\
4 & 15 & 100 & 449 & 0.18100 & $0.07050+0.55940 i$ \\
5 & 10 & 50 & 278 & 0.18146 & $0.07047+0.54295 i$ \\
6 & 20 & 50 & 386 & 0.18089 & $0.06948+0.55924 i$ \\
\hline \hline
\end{tabular}




$$
r=0, \quad 0 \leq z \leq 50: \quad \begin{cases}|m|=0, & \hat{u}=\hat{v}=\partial \hat{w} / \partial r=0, \\ |m|=1, & \hat{u} \pm i \hat{v}=2 \partial \hat{u} / \partial r \pm i \partial \hat{v} / \partial r=\hat{w}=0, \\ |m|>1, & \hat{u}=\hat{v}=\hat{w}=0 .\end{cases}
$$

Here, Eq. (23) was obtained assuming that all physical quantities were smooth and bounded at $r=0$, see Khorrami et $a{ }^{12}$ As in the basic flow case, the pressure at the boundaries is treated implicitly by taking into account the incompressibility condition, given in this case by Eq. (10). Moreover, in order to span a sufficiently large region in the complex $\sigma$ plane, we performed all the computations with three different values of the shift parameter, namely, $\beta=0.2$, $0.2+0.4 i$, and $0.2+0.8 i$. In addition, we used a Krylov subspace of dimension 400 , leading to about $2 \mathrm{~h}$ of computation, for each value of $\beta$, on an Intel ${ }^{\circledR} \mathrm{Xeon}^{\circledR} \mathrm{E} 54302.66 \mathrm{GHz}$ processor.

\section{NUMERICAL RESULTS AND DISCUSSION}

In this section, we present the results obtained from the global stability analysis performed for the geometrical configuration shown in Fig. 1 for a wide range of Reynolds numbers $0 \leq \operatorname{Re} \leq 2200$ and bleed coefficients $0 \leq C_{b} \leq 0.1$, discussing the physical characteristics of the global instabilities observed, as well as the different transitions taking place in the flow. In the particular case without base bleed $C_{b}=0$, the effect of the aspect ratio of the body $L / D$ on the stability properties of the wake will also be briefly addressed.

\section{A. Case without base bleed, $C_{b}=0$}

In this case, where $C_{b}=0$, our calculations indicated that for a body of aspect ratio $L / D=5$, the wake is globally stable for every azimuthal mode $|m|$ in the range $0 \leq \operatorname{Re}<391$. However, for $\operatorname{Re} \simeq 391$ and $|m|=1$ a first bifurcation, whose associated spectrum is shown in Fig. 4(a) marked with a

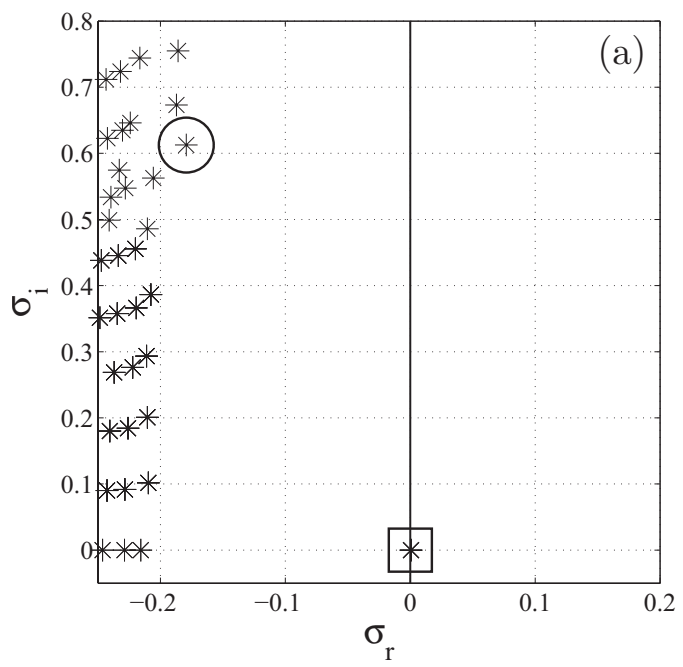

square, takes place. Notice that in this figure, the eigenvalues located into the stable half-plane $\left(\sigma_{r}<0\right)$ have not been filtered to determine their physical or spurious character, since in the present work we are only interested in the unstable eigenvalues $\left(\sigma_{r}>0\right)$. This first bifurcation corresponds to a three-dimensional $(|m|=1)$ and steady $\left(\sigma_{i}=0\right)$ perturbation, whose associated normalized eigenfunctions are plotted in Fig. 5(a), showing a shift in the wake caused by the presence of two counter-rotating streamwise vortices. Moreover, the stability characteristics do not vary significantly in the range of Reynolds numbers $391 \leq \operatorname{Re}<715$, where there is only one unstable mode with $|m|=1, \sigma_{r}>0$, and $\sigma_{i}=0$. However, at $\mathrm{Re} \simeq 715$ a second eigenmode becomes unstable, which corresponds to a three-dimensional $|m|=1$, and oscillating perturbation with a Strouhal number $S_{t}=\sigma_{i} / 2 \pi \simeq 0.0905$. Figure 4(b) shows the eigenvalue spectrum determined at $\mathrm{Re}=715$ and $|m|=1$, where the eigenvalue corresponding to the second bifurcation is marked with a circle. Note in this figure that the eigenvalue associated with the first bifurcation reported above, marked with a square, has now moved into the unstable half-plane since the Reynolds number has been increased. The normalized eigenfunctions obtained at $\mathrm{Re}$ $=715$ and $|m|=1$, and depicted in Fig. 5(b), show the spatially periodic structure associated with this second global mode, which is characteristic of oscillatory wake instabilities. In this figure, only the real part is plotted, the imaginary part being similar but nearly in quadrature with its zeros located approximately at the extrema of the real part. Thus, this mode is a spiral global mode, whereas its counterrotating partner corresponds to the complex conjugate of the eigenvalue. It is worth mentioning that the eigenfunction

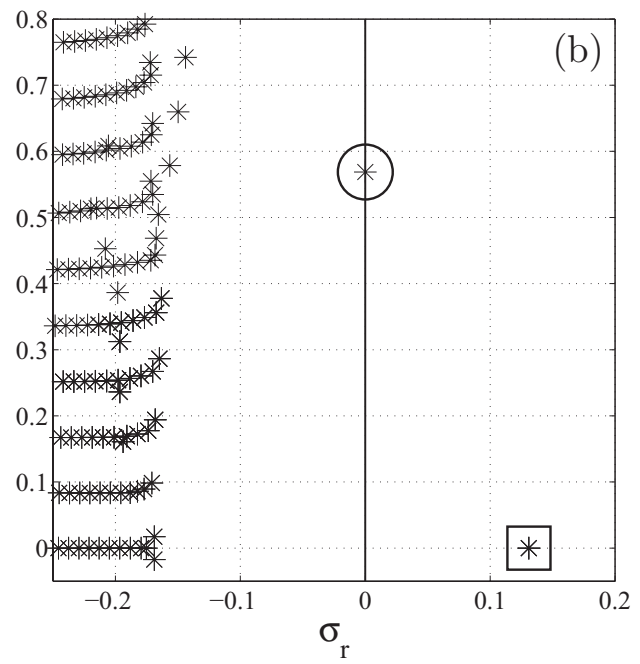

FIG. 4. Eigenvalue spectra for $L / D=5,|m|=1$ and (a) $\operatorname{Re}=391$, (b) $\operatorname{Re}=715$. Squares indicate the eigenvalues corresponding to the first stationary bifurcation $\left(\sigma_{i}=0\right)$ and circles to the second oscillatory bifurcation, whose Strouhal number is $\mathrm{St}=\sigma_{i} / 2 \pi \simeq 0.0905$ in (b). 
(a)

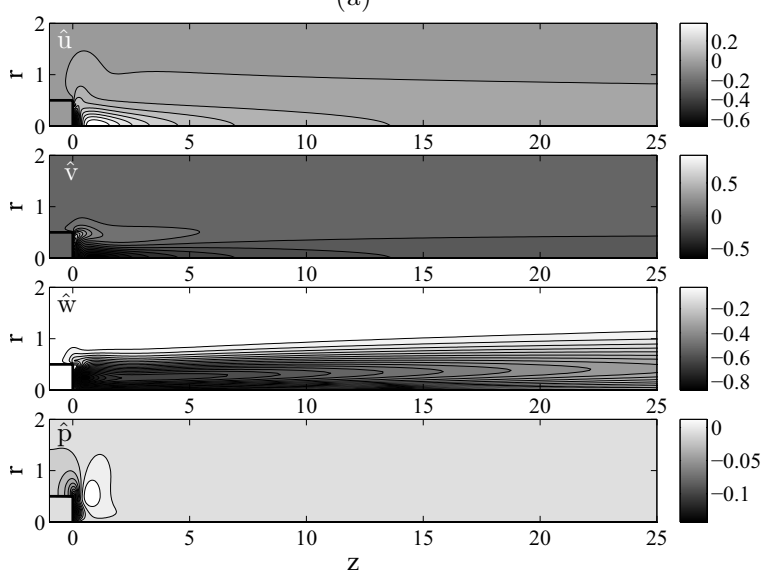

(b)

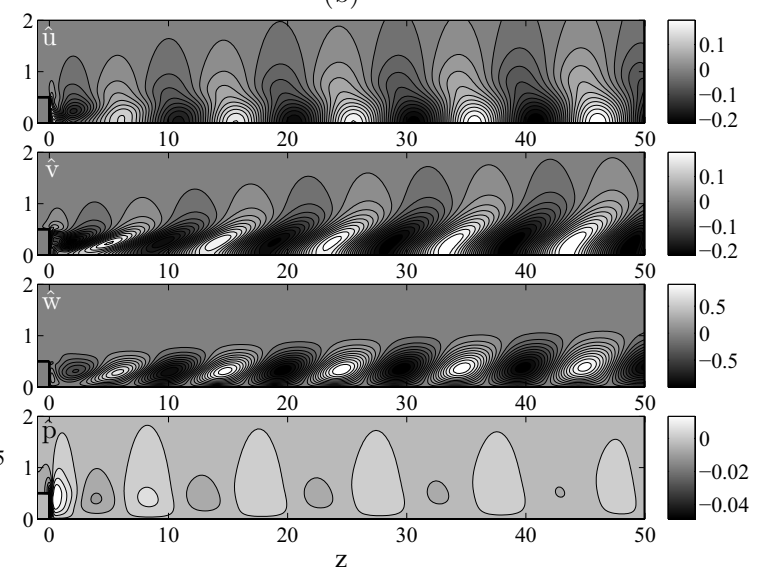

FIG. 5. Isolines of the real part of the normalized eigenfunctions $\hat{u}, \hat{v}, \hat{w}$, and $\hat{p}$ for (a) the eigenvalue marked with a square in Fig. 4(a) and (b) the eigenvalue marked with a circle in Fig. 4(b). Here, the eigenfunctions are normalized with $\|\hat{\mathbf{q}}\|_{\infty}$.

with the largest amplitude in both bifurcations is the one corresponding to the axial velocity component $\hat{w}$.

A study similar to that performed for $|m|=1$ showed that the azimuthal modes $|m|=2$ and $|m|=3$ became unstable at Reynolds numbers higher than those obtained for $|m|=1$, and are, correspondingly, subdominant. However, the azimuthal modes $m=0$ and $|m| \geq 4$ are all stable in the range of Reynolds numbers considered in this work, $0 \leq \operatorname{Re} \leq 2200$.

Let us now briefly describe the effect of the aspect ratio $L / D$ on the critical Reynolds numbers reported above. To that end, we also performed global stability analyses varying $L / D$ from 2 to 10 . The results have been presented in Fig. 6 for $|m|=1$. Notice from Fig. 6(a) that as $L / D$ decreases, the critical Reynolds numbers decrease for both modes, indicating that the wake is more unstable for shorter bodies. This behavior can be explained through the dependence of the basic flow with the aspect ratio at fixed Re. In effect, note that the thickness of the boundary layer at the point of separation decreases as the length of the body decreases, and,
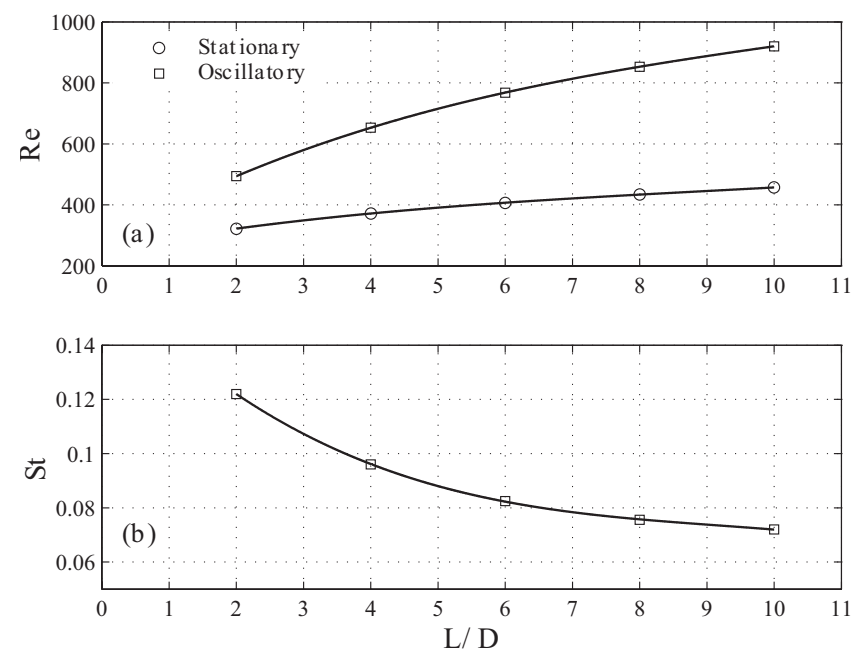

FIG. 6. Dependence of (a) the critical Reynolds number and (b) the Strouhal number on the aspect ratio of the cylinder $L / D$ for $|m|=1$ and without base bleed, $C_{b}=0$. Circles and squares in (a) represent the critical Reynolds numbers for the stationary and the oscillatory bifurcations, respectively. correspondingly, the larger shear stress in the separated boundary layer causes larger values of the recirculating velocity, thus leading to a more unstable flow. The Strouhal number is also affected by changes in $L / D$, as revealed by Fig. 6(b), which shows that the critical frequency of the oscillating mode decreases as the length of the body increases.

To check the accuracy of our calculations, we have compared the values of the critical Reynolds number and the Strouhal number for the second bifurcation obtained for a truncated body of aspect ratio $L / D=4$, namely, $\mathrm{St} \simeq 0.095$ and $\operatorname{Re} \simeq 659$ (see Fig. 6), with those reported by Meliga et al. ${ }^{13}$ obtained for the same geometry but using a numerical code based on a finite-element spatial discretization, $\mathrm{St}$ $\simeq 0.09$ and $\mathrm{Re} \simeq 666$. Note that both results are nearly identical in spite of the different numerical methods used.

\section{B. Case with base bleed, $C_{b}>0$}

As already mentioned in Sec. I, base bleed is a simple control mechanism proposed to inhibit the periodic emission of vortices in the wake behind bluff bodies occurring at supercritical Reynolds numbers. In this section, we report the results provided by the global stability analyses performed for a fixed geometry, a truncated body of aspect ratio $L / D$ $=5$, when a stream of fluid of the same density and viscosity as the ambient fluid is blown throughout the body base, $C_{b}>0$.

To evaluate the effect of the base bleed on both the eigenvalue spectra and the eigenfunctions, let us show the results obtained for two different cases: (a) a flow unstable only to the first steady mode $(391<\operatorname{Re}=500<715,|m|$ $=1$ ) and (b) a case supercritical with respect to both the steady and the oscillatory global instabilities $(715<\mathrm{Re}$ $=1000,|m|=1)$. In Fig. 7 we have plotted the spectra for $\operatorname{Re}=500,|m|=1$, and different values of $C_{b}$. Figure 7(a) displays the spectrum for $C_{b}=0$, which shows the existence of a single unstable eigenvalue. As the bleed coefficient is slightly increased, for instance to $C_{b}=0.020$ corresponding to Fig. 7(b), the growth rate of the unstable mode decreases, as this unstable eigenvalue moves toward the stable half-plane. This trend continues for increasing values of $C_{b}$ until a criti- 

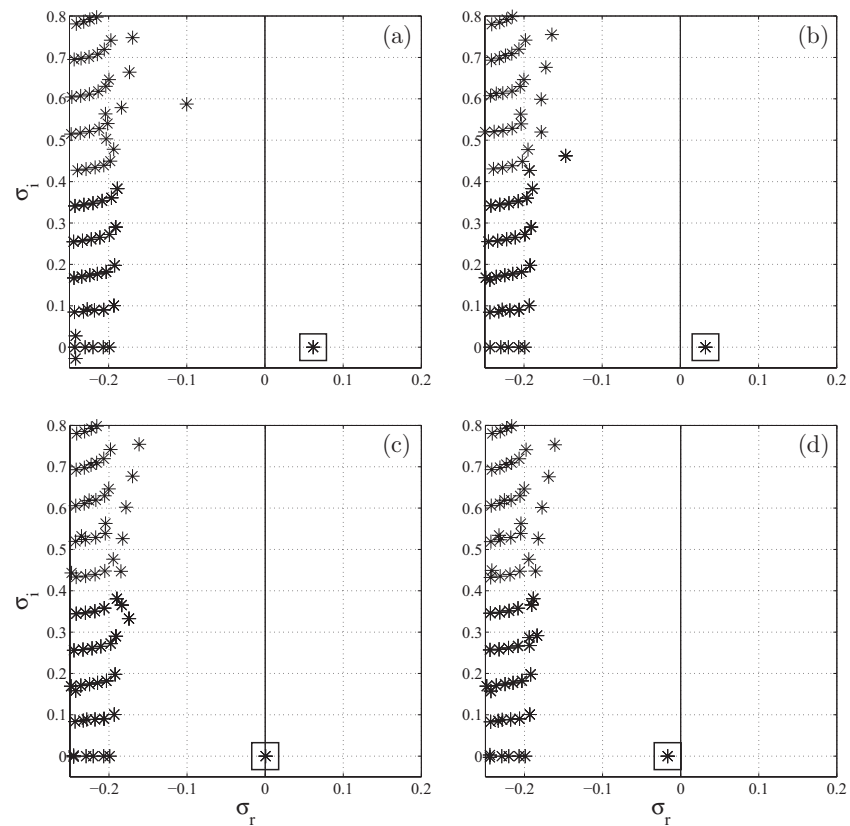

FIG. 7. Eigenvalue spectra for $L / D=5, \operatorname{Re}=500$, and $|m|=1$ and base bleed coefficients (a) $C_{b}=0$, (b) $C_{b}=0.020$, (c) $C_{b}=C_{b 1}^{*}=0.040$, and (d) $C_{b}$ $=0.050$, respectively.

cal bleed coefficient is eventually found, $C_{b}=C_{b 1}^{*}(\mathrm{Re}$ $=500,|m|=1) \simeq 0.040$, for which the instability becomes neutral, as shown in Fig. $7(\mathrm{c})$. Finally, for $C_{b}>C_{b 1}^{*}$ all the eigenvalues are located within the stable half-plane, as displayed in Fig. $7(\mathrm{~d})$, where $C_{b}=0.050$. Similarly, Fig. 8 shows the eigenvalue spectra obtained for $\operatorname{Re}=1000,|m|=1$, and different values of $C_{b}$. Notice that, when $C_{b}=0$, there are two unstable modes, one steady and one oscillatory. However, as
$C_{b}$ increases, the oscillating mode is stabilized first when $C_{b}>C_{b 2}^{*}=0.030$, as can be seen in Fig. 8(c). For larger values of $C_{b}$, the steady mode becomes also stable when $C_{b}$ $>C_{b 1}^{*}=0.082$ in this case, as shown in Fig. 8(f). Furthermore, the spatial structure of the global modes is also affected by the presence of base bleed, as revealed by an examination of the eigenfunctions $\hat{w}$ shown in Fig. 9, associated with both the steady and the oscillatory unstable modes displayed in Fig. 8. Note that the most prominent effect of base bleed is to push the eigenfunctions downstream from the base, and, in the case of the unsteady global mode, to considerably increase the wavelength of the perturbation. This increase is corresponded by a decrease in the frequency $\sigma_{i}$, as can be observed in Fig. 8.

Finally, the Re- $C_{b}$ parameter plane shown in Fig. 10(a) summarizes the different instability regimes found for the flow configuration under study here. In Fig. 10(a), the solid line represents the evolution with the Reynolds number of the critical bleed coefficient required to stabilize the steady instability for the azimuthal modes $|m|=1, C_{b 1}^{*}=C_{b 1}^{*}(\operatorname{Re},|m|$ $=1$ ), and the dashed line corresponds to the critical values of $C_{b}$ needed to stabilize the oscillatory mode, $C_{b 2}^{*}$ $=C_{b 2}^{*}(\operatorname{Re},|m|=1)$. Notice that $C_{b 1}^{*}>C_{b 2}^{*}$ for all Reynolds numbers studied, indicating that the oscillatory mode is always the first to be stabilized for increasing values of $C_{b}$. Let us briefly discuss the different regions identified in the stability plane displayed in Fig. 10(a). In region I, which corresponds to $C_{b}>C_{b 1}^{*}$, the axisymmetric steady wake flow is linearly stable for all azimuthal numbers $m$. However, in region II, for which $C_{b 2}^{*} \leq C_{b} \leq C_{b 1}^{*}$, only the steady $|m|=1$ global mode is unstable, indicating that a steady threedimensional wake structure is to be expected in experiments
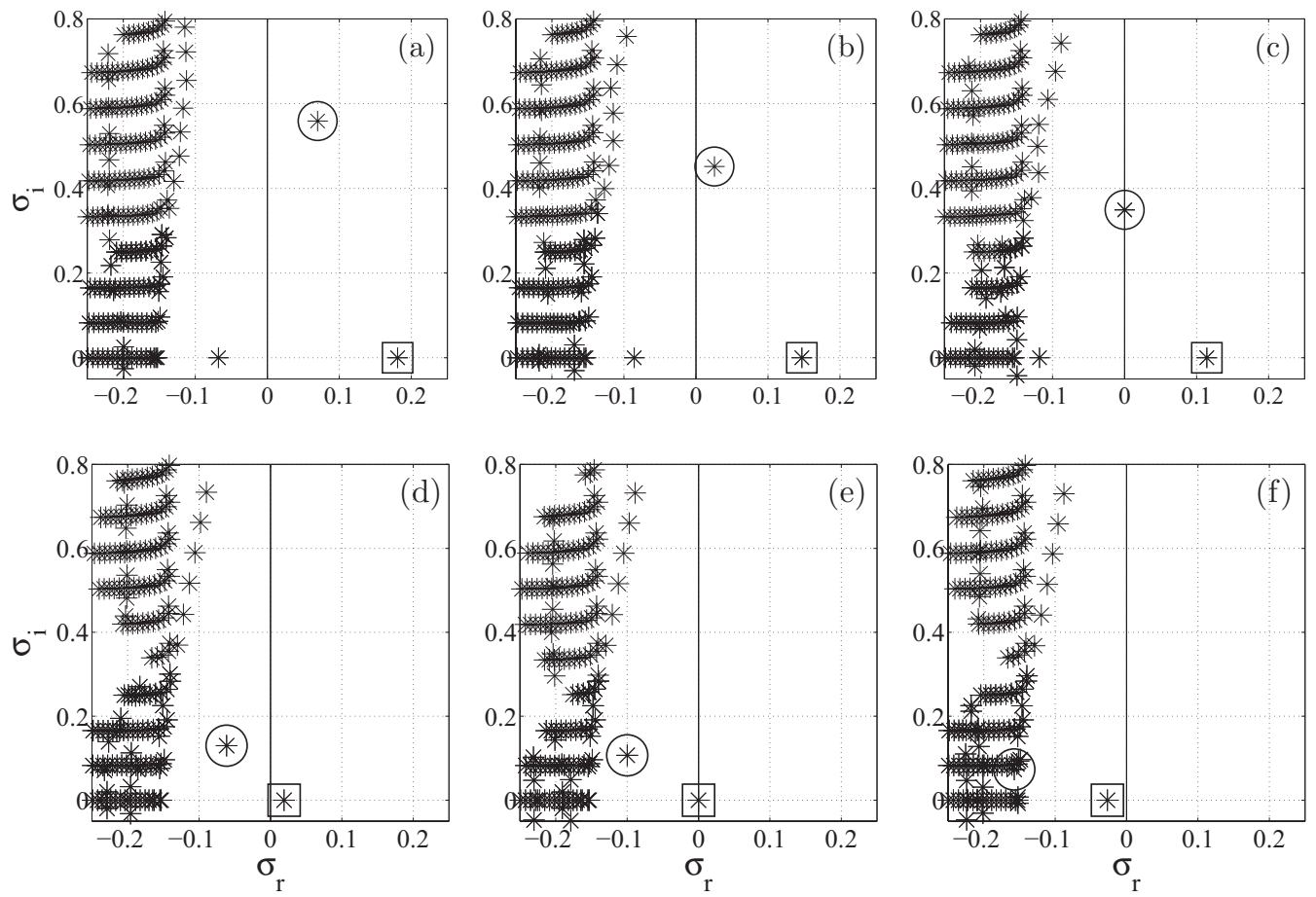

FIG. 8. Eigenvalue spectra for $L / D=5, \mathrm{Re}=1000$, and $|m|=1$ and base bleed coefficients (a) $C_{b}=0$, (b) $C_{b}=0.015$, (c) $C_{b}=C_{b 2}^{*}=0.030,(\mathrm{~d}) C_{b}=0.075$, (e) $C_{b}=C_{b 1}^{*}=0.082$, and (f) $C_{b}=0.090$, respectively. 

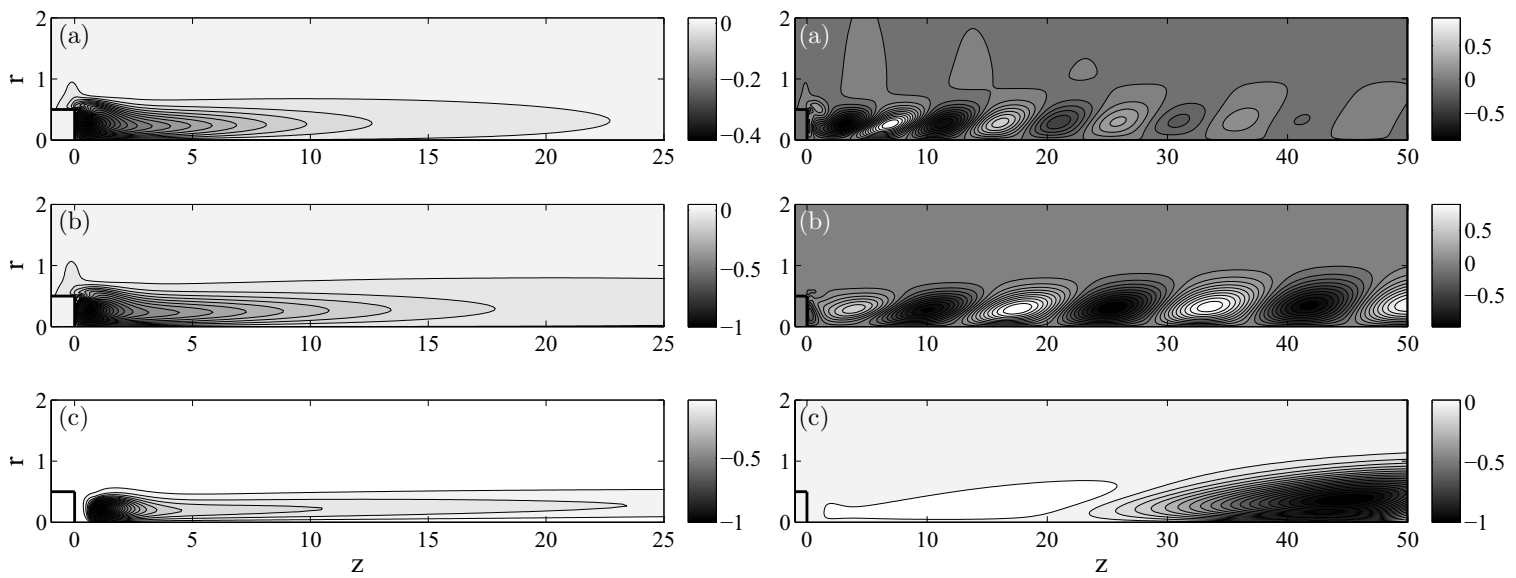

FIG. 9. Isolines of the real part of the normalized eigenfunction $\hat{w}$ for $L / D=5, \operatorname{Re}=1000$, and $|m|=1$ and (a) $C_{b}=0$, (b) $C_{b}=0.030$, and (c) $C_{b}=0.082$, respectively. The left column shows the effect of base bleed on the first stationary bifurcation mode and the right column on the second oscillatory bifurcation.

performed within this region. Finally, in region III, for which $C_{b}<C_{b 2}^{*}$, both the steady and the oscillatory global modes are unstable for the azimuthal number $|m|=1$ and, consequently, periodic behavior is expected to take place within this region, at least in the vicinity of the critical curve, where nonlinear effects are small. Although not shown in Fig. 10(a) for clarity, the azimuthal modes $|m|=2$ and $|m|=3$ can also become unstable inside region III depending on the values of Re and $C_{b}$, but their growth rates are always far below those of the helical mode $|m|=1$, and are, correspondingly, subdominant.

The dependence of the Strouhal number on $C_{b}$ for different values of the Reynolds number is also shown in Fig. 10(b). Note that St is nearly independent of the Reynolds number when $C_{b}=0$, and it decreases with $C_{b}$ for all Reynolds numbers considered, as already displayed in Fig. 8 for the frequency $\sigma_{i}$. In Fig. 10(b), the end point for each Re corresponds to the critical bleed coefficient for which the oscillatory mode restabilizes, $C_{b 2}^{*}$.

\section{EXPERIMENTAL RESULTS}

The experiments were performed in an open low turbulence intensity (lower than $1.5 \%$ ) wind tunnel of 20 $\times 20 \mathrm{~cm}^{2}$ square test section. An aluminum cylindrical body of $1 \mathrm{~cm}$ of diameter and total length of $2 \mathrm{~cm}$ with an ellipsoidal rounded nose, of 2:1 major-to-minor axis ratio, was introduced at the centerline of the wind tunnel, aligned with the direction of the free stream. The body was held from its center of gravity by a needle of external diameter $1 \mathrm{~mm}$. The fluctuations of the streamwise component of the velocity were recorded using a hot wire placed seven diameters downstream from the base of the body, a distance where the amplitude of the velocity fluctuations was found to be the largest.

For Reynolds numbers above the critical one, $\mathrm{Re}_{c o}$, the streamwise velocity time trace displays periodic oscillations with a characteristic frequency corresponding to the

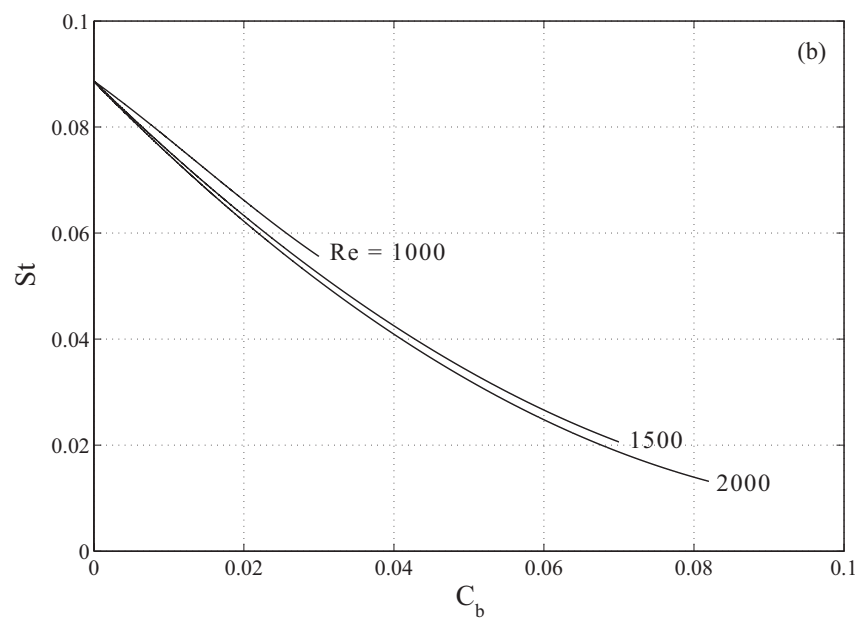

FIG. 10. (a) Evolution of the critical base bleed coefficients with the Reynolds number for the truncated cylinder with aspect ratio $L / D=5$ and the most unstable azimuthal mode $|m|=1$. Solid and dashed lines correspond to the critical bleed coefficients needed to stabilize the stationary $C_{b 1}^{*}$ and oscillatory $C_{b 2}^{*}$ global modes, respectively. (b) Evolution of the Strouhal number St with the base bleed coefficient $C_{b}$ for Reynolds numbers $\operatorname{Re}=1000, \operatorname{Re}=1500$, and $\mathrm{Re}$ $=2000$. The end point for each Re corresponds to the critical bleed coefficient $C_{b 2}^{*}$. 

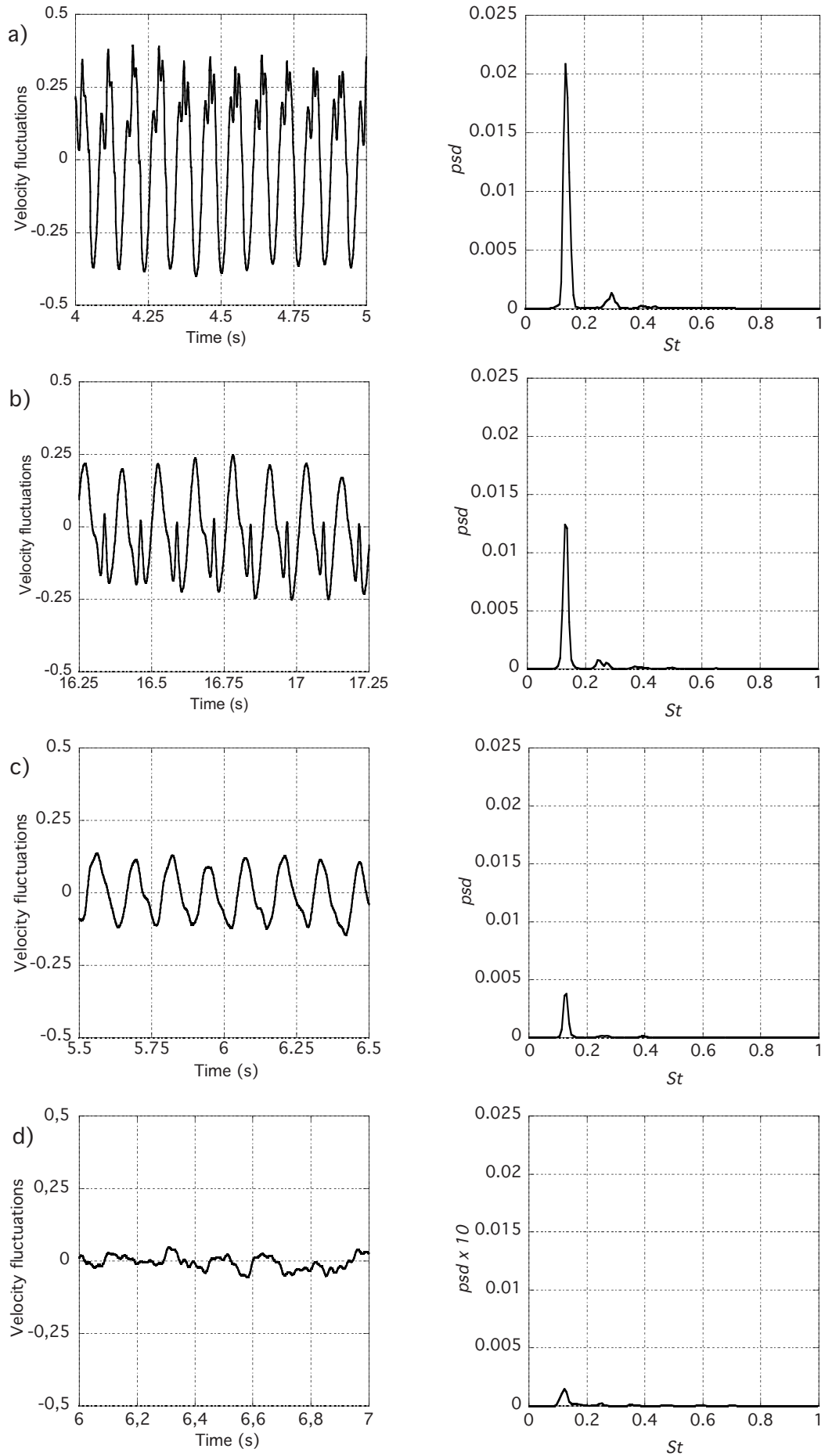

FIG. 11. Hot-wire measurements of the fluctuations of the streamwise velocity $w^{\prime}(t)$ and the corresponding power spectral density at $(r=0, z=7)$ behind the body base for (a) $\operatorname{Re}=493$, (b) $\operatorname{Re}=443$, (c) $\operatorname{Re}=411$, and (d) $\operatorname{Re}=386$, respectively. Notice that the energy spectrum at $\operatorname{Re}=386$ has been multiplied by a factor of 10 in (d). shedding frequency observed in the power spectral density [see Fig. 11(a) where $\mathrm{Re}=493$ ]. The power spectral density shown in Fig. 11(a) also presents lower amplitude peaks corresponding to harmonics of the characteristic frequency. When the Reynolds number is decreased, the amplitude of the oscillations also decreases, as shown in Fig. 11(b), for $\operatorname{Re}=443$. Notice in this figure that the amplitude of the oscillations is lower than that shown in Fig. 11(a) and, therefore, the energy associated with the shedding frequency displayed in the corresponding spectrum decreases as well. In this case, the energy spectrum also displays a sharp peak, associated with the shedding frequency corresponding to this
Reynolds number together with its harmonics. Figures 11(a) and 11(b) present a modulation of the velocity signal which may be caused by the loss of planar symmetry of the wake, as already reported by Schouveiler and Provansal ${ }^{14}$ in the wake of a sphere. As the Reynolds number decreases further, the amplitude of the oscillations keeps decreasing until $\mathrm{Re}$ becomes smaller than $\mathrm{Re}_{c o}$ where the periodic behavior disappears [see Fig. 11(d) where $\mathrm{Re}=386$ ].

To determine the critical value of the Reynolds number $\operatorname{Re}_{c o}$, we have represented in Fig. 12 the evolution with the Reynolds number of the amplitude of the streamwise velocity fluctuations $w_{f_{c}}^{\prime 2}$ determined as 


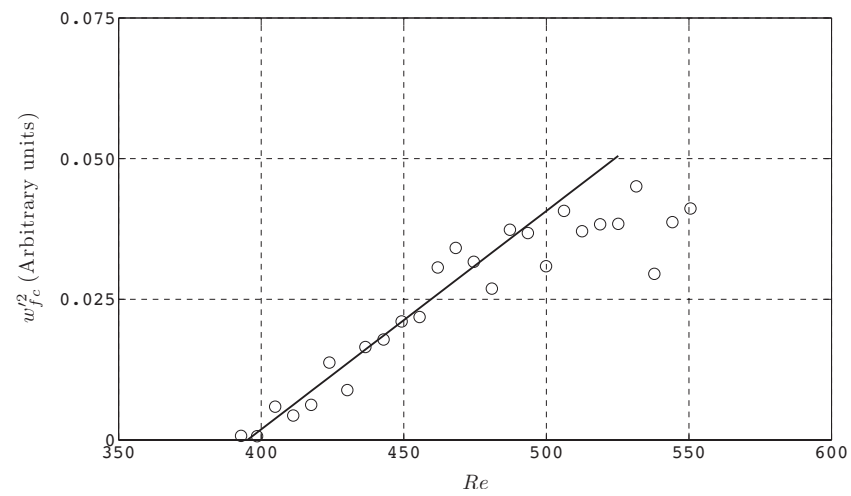

FIG. 12. Squared amplitude of the streamwise velocity perturbations $w_{f_{c}}^{\prime 2}$ at $(r=0, z=7)$ behind the base of the body vs the Reynolds number.

$$
w_{f_{c}}^{\prime 2}=\int_{f_{c}-\Delta f_{d}}^{f_{c}+\Delta f_{u}} \operatorname{psd}(f) d f,
$$

where $\operatorname{psd}(f)$ is the power spectral density obtained from the velocity signals, $f_{c}$ is the characteristic shedding frequency, and $\Delta f_{d}$ and $\Delta f_{u}$ correspond to the interval of frequency around $f_{c}$ for which the power spectral density drops down to $5 \%$ of the peak value, $\operatorname{psd}\left(f_{c}+\Delta f_{u}\right)=\operatorname{psd}\left(f_{c}-\Delta s f_{d}\right)$ $=0.05 \operatorname{psd}\left(f_{c}\right)$. Thus, $w_{f_{c}}^{\prime 2}$ represents the narrow-band energy at the peak frequency $f_{c}$ evaluated from the energy spectrum. The linear increase in $w_{f_{c}}^{\prime 2}$ with the Reynolds number near the critical value indicates that the transition to the oscillatory regime corresponds to a Hopf bifurcation. The critical value of the Reynolds number was then determined by linear regression of the experimental measurements of $w_{f_{c}}^{\prime 2}$ near criticality, providing $\operatorname{Re}_{c o}^{\text {expt }}=395$, with a corresponding Strouhal number of $\mathrm{St}_{c}^{\mathrm{expt}}=0.117$.

To perform the global stability analysis with the spectral numerical code described above, preliminary simulations of the basic flow were carried out by a standard finite volume method of second order of precision for the velocity and pressure using the SIMPLE algorithm. ${ }^{15}$ The axisymmetric computational domain used in these preliminary simulations was $-12 \leq z \leq 50$ and $0 \leq r \leq 10$ [a detail of the grid near the body can be observed in Fig. 13(a)]. The final basic flow used in the linear stability study was obtained with the spec-

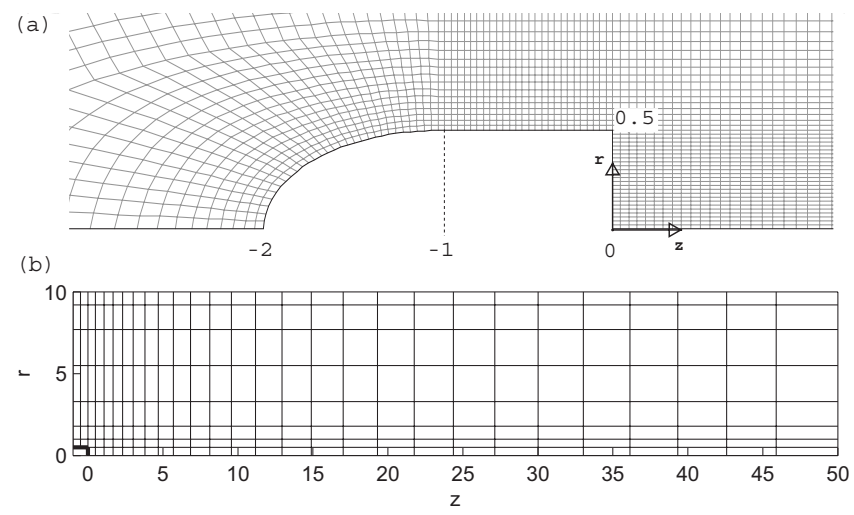

FIG. 13. (a) Detail of the grid used to obtain the basic flow with the finite volume code. (b) Spectral element mesh for $z \geq-1$. tral method described in Sec. II A, for a grid domain given by $-1 \leq z \leq 50$ and $0 \leq r \leq 10$, using the preliminary finite volume simulations as initial conditions for the NewtonRaphson method. Consequently, only the inlet condition at $z=-1$ was obtained from the finite-volume computations in order to precisely reproduce the experimental conditions. The mesh used for the global stability study, which consists of 222 subdomains, is shown in Fig. 13(b). A grid convergence study, similar to that described in Sec. II, was performed, concluding that the optimal value of the polynomial order was $n=11$ with a total number of spectral nodes equal to 27259 . The final outlet length used was $z=50$ for which the spectra did not change, and the boundary conditions were those described in Sec. II, with the difference that nonslip conditions were imposed at $r=10$ to reproduce the wall conditions of the experiments performed in the wind tunnel.

The critical Reynolds number obtained experimentally for the oscillatory bifurcation was $\mathrm{Re}_{c o}^{\text {expt }}=395$ with an associated Strouhal number of $\mathrm{St}_{c}^{\text {expt }}=0.117$, while the stability analysis gives a transitional Reynolds number for the oscillatory bifurcation of $\mathrm{Re}_{c o}^{\text {num }}=518$ with a corresponding Strouhal number of $\mathrm{St}_{c}^{\text {num }}=0.102$, and a critical Reynolds number for the stationary bifurcation of $\operatorname{Re}_{c s}^{\text {num }}=327$. Notice that the relative errors in the numerical predictions are $31 \%$ for the oscillatory bifurcation and $14.7 \%$ for the Strouhal number at the transitional Reynolds number. Note also that the values of the critical Reynolds numbers obtained for the bulletshaped body are only slightly larger than those obtained for the truncated cylinder with the same aspect ratio $L / D=2$ reported in Sec. III A, namely, $\operatorname{Re}_{c s} \simeq 322$ and $\operatorname{Re}_{c o} \simeq 494$ for the steady and oscillatory global modes, respectively (see Fig. 6). The small differences, which are $1.5 \%$ for the steady bifurcation and lower than $5 \%$ for the oscillatory Reynolds number, can be attributed to the slight differences in the boundary layer thickness at the trailing edge and, thus, the results are expected to be nearly identical for larger values of the body's aspect ratio.

Both, the experimental and the numerical results obtained for the evolution of the Strouhal number St versus the Reynolds number Re are shown in Fig. 14. As can be observed in this figure, the Strouhal number measured experimentally grows slightly with the Reynolds number from St $=0.117$ at $\mathrm{Re}=399$ to $\mathrm{St}=0.153$ at $\mathrm{Re}=570$, whereas the value of the numerical Strouhal number, $\mathrm{St} \simeq 0.102$, barely varies with Re. The disagreement between experiments and stability analysis in the values of the critical Reynolds number and of the associated Strouhal number is not surprising since, after the first steady bifurcation at $\operatorname{Re}_{c s}=327$, the flow loses the axisymmetry. In effect, the steady $|m|=1$ perturbation is believed to saturate at a finite amplitude which increases with $\mathrm{Re}$ and should be large at the second critical Reynolds number, $\operatorname{Re}_{c o}=518$, where the axisymmetric mode starts to be unstable to the oscillatory $|m|=1$ modes. This behavior is in contrast with that of the sphere and disk wakes, ${ }^{16-18}$ where the predictions of the stability analysis performed on the axisymmetric basic flow, already unstable to a steady $m=1$ global mode, are much closer to the experiments than in the case considered here. This behavior of the sphere and disk wakes has been recently explained by Fabre 


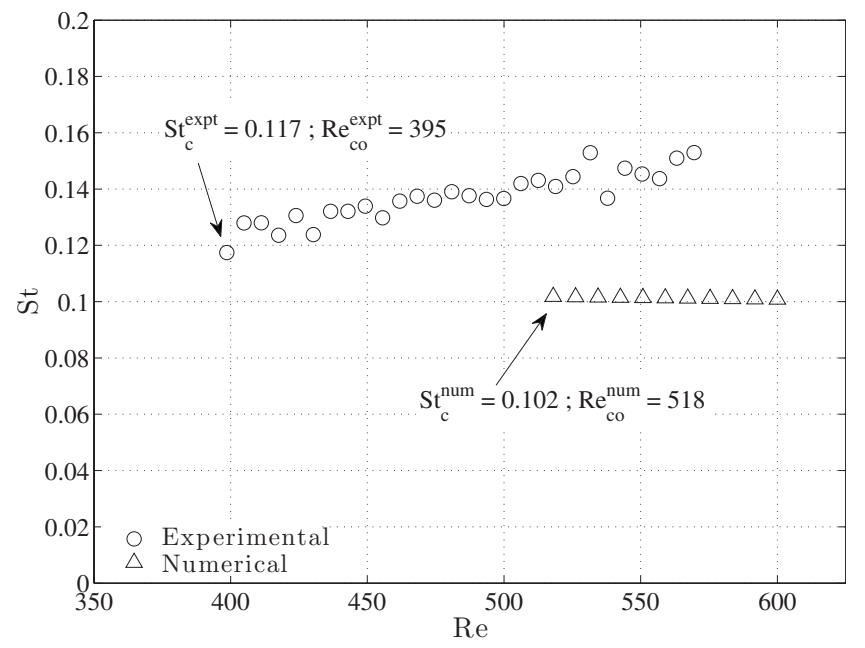

FIG. 14. Experimental and numerical evolution of the Strouhal number St with the Reynolds number Re for the bulletlike body of aspect ratio $L / D$ $=2$ shown in Fig. 13(a).

et al. ${ }^{19}$ using a normal form theory, who have shown that the bifurcated steady $|m|=1$ branch of finite amplitude is destabilized by a resonant interaction between the $m=1$ and the $m=-1$ oscillatory modes of the axisymmetric basic flow resulting in a secondary Hopf bifurcation occurring at a slightly lower Reynolds number than the critical one corresponding to the axisymmetric flow. However, this is not the case for the geometry considered in this work, where the secondary bifurcation takes place at $\operatorname{Re}_{c o}^{\text {expt }}=395$, considerably smaller than the value corresponding to the destabilization of the axisymmetric flow by oscillating modes at $\operatorname{Re}_{c o}^{\text {num }}=518$. Concerning this point, it is worth mentioning that the difference between the critical Reynolds numbers associated with the steady and oscillatory modes decreases as $L / D$ decreases, as revealed by Fig. 6 . Thus, the differences between the stability properties of the wake of a truncated cylinder, considered in this work, and those of the wakes of spheres and disks, discussed above, are expected to become more pronounced as $L / D$ increases. According to the theory of Fabre et al., ${ }^{19}$ the Strouhal number at the secondary bifurcation is close to the Strouhal number associated with the oscillating mode, and this prediction seems to be in better agreement with the present comparison of the experiments to the stability theory, which gives a difference of $14.7 \%$ in Strouhal number.

However, notice that the normal form theory developed by Fabre et al. ${ }^{19}$ is based on the assumption that the different modes interacting in the slightly supercritical wake must be simultaneously nearly neutral, i.e., that the critical Reynolds numbers of the first (steady) and second (oscillatory) eigenmodes bifurcating from the basic axisymmetric flow must be close to each other. Furthermore, Fabre et al. ${ }^{19}$ propose $\left(\operatorname{Re}_{c o}-\operatorname{Re}_{c s}\right) / \operatorname{Re}_{c s}$ as a measure of the difference in the critical Reynolds numbers of the two bifurcations and argue that this difference must be small for the normal form theory to apply. Although $\left(\operatorname{Re}_{c o}-\operatorname{Re}_{c s}\right) / \operatorname{Re}_{c s} \ll 1$ in the case of the sphere and disk wakes, it has the value (518-327)/327 $\simeq 0.584$ for our bulletlike geometry and, unlike what hap- pens in the sphere and disk wakes, the validity of the normal form theory developed by Fabre et al. is questionable for the geometry under consideration in this work. Thus, both a more complete experimental study and three-dimensional direct numerical simulations are needed to check the predictions of the normal form theory in the wake of slender bodies with a blunt trailing edge.

\section{CONCLUSIONS}

We have performed a global stability analysis of the incompressible, laminar, and axisymmetric flow around a slender body with a blunt trailing edge, allowing us to characterize the instabilities appearing in its wake. The analysis also contemplates the use of a stream of fluid injected from the base of the body, or base bleed, as a possible mechanism to control these wake instabilities, as already considered in Sevilla and Martínez-Bazán ${ }^{5}$ in the case of turbulent wakes at larger values of $\mathrm{Re}$ than those considered here.

We first analyzed the case of a truncated cylinder with aspect ratio $L / D=5$, aligned with the flow and uniform velocity profile at the inlet. For this particular geometry, and in the case without base bleed, the axisymmetric steady flow has been shown to be stable for $\operatorname{Re} \lesssim 391$. At this critical value of $\mathrm{Re}$, a steady global mode with azimuthal number $|m|=1$ becomes unstable, and the examination of the associated eigenfunctions shows that the bifurcation has a structure which resembles the first bifurcation occurring in the case of a sphere, ${ }^{16,17}$ and which leads to the double-threaded wake structure observed in experiments. ${ }^{14}$ For larger values of Re, our calculations reveal the existence of a second Hopf bifurcation of the steady axisymmetric wake at $\mathrm{Re} \simeq 715$, for which an oscillatory global mode with $|m|=1$ and St $\simeq 0.0905$ becomes unstable. Again, the eigenfunctions for this mode are similar to those observed in the case of the second bifurcation for the sphere wake ${ }^{16,17}$ and correspond to a couple of counter-rotating, spatially periodic helical waves. In the nonlinear regime observed in unforced experiments, this couple of helical waves leads to the shedding of streamwise-oriented, alternating hairpinlike vortices. ${ }^{14,20}$ The effect of the aspect ratio of the cylinder was also addressed by performing several computations within the range 2 $\leq L / D \leq 10$, which revealed that the effect of $L / D$ is quantitative but not qualitative: larger values of $L / D$ lead to more stable wakes in the sense that the critical values of the Reynolds number of both the steady and oscillatory $|m|=1$ bifurcations increase as $L / D$ increases. In the case of the oscillatory mode, the critical value of the Strouhal number was shown to decrease slightly as $L / D$ increases.

In the case with base bleed, our results show the existence of two critical values of the bleed coefficient $C_{b}$, defined here as the bleed-to-free-stream velocity ratio. For $C_{b}$ $>C_{b 1}^{*}(\operatorname{Re},|m|)$, the steady eigenmode becomes stable, while for $C_{b}>C_{b 2}^{*}=C_{b 2}^{*}(\operatorname{Re},|m|)$, such that $C_{b 1}^{*}>C_{b 2}^{*}$, the oscillatory one is stabilized. Moreover, for supercritical Reynolds numbers, $\operatorname{Re}>\operatorname{Re}_{c s}=391$, the whole eigenvalue spectrum moves toward the stable half-plane as $C_{b}$ increases, indicating the stabilizing properties of base bleed. In addition, when 
$\operatorname{Re}>\operatorname{Re}_{c o}=715$, as $C_{b}$ increases the Strouhal number of the oscillatory mode decreases, and its associated wavelength increases.

We have also performed hot-wire measurements in the wake of a cylindrical body with aspect ratio $L / D=2$ aligned with the free stream, and rounded at the nose to avoid separation of the boundary layer. The experiments reveal the existence of a Hopf bifurcation in the wake at a critical value $\mathrm{Re}_{c o}^{\text {expt }} \simeq 395$, corresponding to the transition to a periodic wake with Strouhal number $\mathrm{St}_{c}^{\text {expt }} \simeq 0.117$ at criticality, and which increases slightly with $\operatorname{Re}$ for $\operatorname{Re}>\operatorname{Re}_{c o}^{\text {expt }}$. These results show some discrepancies with the linear, global stability analysis of the basic flow computed for the geometry used in the experiments, which gives a transition to the oscillatory mode at a higher critical Reynolds number $\operatorname{Re}_{c o}^{\text {num }}$ $\simeq 518$ with associated Strouhal number $\mathrm{St}_{c}^{\text {num }} \simeq 0.102$. The significant differences found between the experiments and the stability analysis are due to the limitations inherent in using an axisymmetric basic flow for Reynolds numbers higher than the one corresponding to the first bifurcation, for which the original basic flow is already perturbed and, in particular, is no longer axisymmetric due to the threedimensional nature of the first bifurcation $(|m|=1$ mode). This discrepancy in the prediction of the second bifurcation between the experiments and the stability analysis shows that a more elaborate approach is needed to precisely characterize the unsteady instabilities appearing in the wake of a slender body with a blunt trailing edge. Therefore, three-dimensional direct numerical simulations, as well as a more complete experimental characterization of the wake, need to be performed.

Notice that the values obtained for the critical Reynolds number and for the Strouhal number depend on the geometry of the body through changes in the basic flow, as clearly shown by the results discussed above. For instance, for a given value of $\mathrm{Re}$, the thickness of the boundary layer separating from the trailing edge of the body depends on the aspect ratio $L / D$. In turn, as shown in Sec. III, the thickness of the boundary layer is known to affect the properties of the resulting basic flow: thinner boundary layers provide larger recirculation velocities in the separation bubble and, thus, more unstable wakes. Correspondingly, the critical Reynolds numbers for the stationary and the oscillatory bifurcations, $\operatorname{Re}_{c s}$ and $\operatorname{Re}_{c o}$, respectively, increase as $L / D$ increases, in agreement with the results computed here in the range of aspect ratios $2 \leq L / D \leq 10$. This reason, together with the fact that the wake is laminar for the values of Re considered in this work, also explains the lower values computed here for the critical bleed coefficient compared with those obtained in Sevilla and Martínez-Bazán, ${ }^{5}$ where the authors employed an upstream velocity profile with a boundary layer much thinner than those considered in this work, and where the Reynolds number used was also larger than those studied here.

Finally, although the linear global approach is justified near the first bifurcation and, consequently, can be correctly used to determine the associated value of the critical bleed coefficient $C_{b 1}^{*}$, it is important to emphasize the limitations of the techniques employed in this work. For instance, our com- putation of the second bifurcation employs the steady axisymmetric wake as basic flow, which is already unstable to the first bifurcation and, thus, is not expected to represent the right flow field present in real situations at threshold, as has already been remarked above. A rigorous calculation of the second bifurcation, and of the associated value of $C_{b 2}^{*}$, would require a global stability analysis of the steady flow resulting from the saturation of the first bifurcation. However, the recent work by Fabre et al. ${ }^{19}$ has established the link between the successive bifurcations of the real flow and the stability properties of the axisymmetric basic flow. Extrapolating their results, a base bleed that stabilizes the oscillating perturbations of the axisymmetric flow should also stabilize the oscillating perturbation of the bifurcated flow. Amazingly, for high enough Reynolds numbers one may imagine that without base bleed the flow results solely from the saturation of the oscillating mode with no contribution of the stationary $m=1$ mode. Increasing the base bleed will restabilize the oscillating mode and the stationary mode should then reappear, since our computations show that it needs a larger bleed coefficient to restabilize. Moreover, increasing the base bleed makes us go backward through the sequence of bifurcations predicted by the generic normal form of Fabre et al. ${ }^{19}$ In practice, the lift of the body should evolve from an oscillating lift with zero mean value to an oscillating lift with a finite mean value, and then to a steady lift before reaching a zero lift when all modes have been restabilized. The experimental measurements being prepared will, in particular, test such prediction.

\section{ACKNOWLEDGMENTS}

This research was supported by the Spanish MCyT and Junta de Andalucía under Project Nos. DPI2008-06624C03-02 and P07-TEP02693, respectively. E.S.R. also wants to acknowledge the help and hospitality of the staff at LadHyx (Ecole Polytechnique of Paris) during his research stay, as well as the support of the Ramón y Cajal program.

${ }^{1}$ A. Zebib, "Stability of viscous flow past a circular cylinder," J. Eng. Math. 21, 155 (1987).

${ }^{2}$ P. A. Monkewitz and L. N. Nguyen, "Absolute instability in the near-wake of two-dimensional bluff bodies," J. Fluids Struct. 1, 165 (1987).

${ }^{3}$ J.-M. Chomaz, "Global instabilities in spatially developing flows: Nonnormality and nonlinearity," Annu. Rev. Fluid Mech. 37, 357 (2005).

${ }^{4}$ H. Choi, W.-P. Jeon, and J. Kim, "Control of flow over a bluff body," Annu. Rev. Fluid Mech. 40, 113 (2008).

${ }^{5}$ A. Sevilla and C. Martínez-Bazán, "Vortex shedding in high Reynolds number axisymmetric bluff-body wakes: Local linear instability and global bleed control," Phys. Fluids 16, 3460 (2004).

${ }^{6}$ M. O. Deville, P. F. Fischer, and E. H. Mund, High Order Methods for Incompressible Fluid Flow (Cambridge University Press, Cambridge, England, 2002).

${ }^{7}$ D. A. Knoll and D. E. Keyes, "Jacobian-free Newton-Krylov methods: A survey of approaches and applications," J. Comput. Phys. 193, 357 (2004).

${ }^{8}$ C. Canuto, M. Y. Hussaini, A. Quarteroni, and T. A. Zang, Spectral Methods in Fluid Dynamics (Springer-Verlag, Berlin, 1991).

${ }^{9}$ R. Peyret, Spectral Methods for Incompressible Viscous Flow (SpringerVerlag, Berlin, 2002).

${ }^{10} \mathrm{U}$. Ehrenstein and F. Gallaire, "On two-dimensional temporal modes in spatially evolving open flows: The flat-plate boundary layer," J. Fluid Mech. 536, 209 (2005).

${ }^{11}$ D. S. Henningson and E. Åkervik, "The use of global modes to understand transition and perform flow control," Phys. Fluids 20, 031302 (2008). 
${ }^{12}$ M. R. Khorrami, M. R. Malik, and R. L. Ash, "Application of spectral collocation techniques to the stability of swirling flows," J. Comput. Phys. 81, 206 (1989).

${ }^{13}$ P. Meliga, D. Sipp, E. Garnier, and J. M. Chomaz, "A theoretical study of the frequency selection mechanisms in afterbody unsteadiness," Proceedings of the 37th AIAA Fluid Dynamics Conference and Exhibit, Miami (AIAA, Reston, VA, 2007), Paper No. 2007-3977.

${ }^{14}$ L. Schouveiler and M. Provansal, "Self-sustained oscillations in the wake of a sphere," Phys. Fluids 14, 3846 (2002).

${ }^{15}$ J. H. Ferziger and M. Perić, Computational Methods for Fluid Dynamics (Springer-Verlag, Berlin, 2002).
${ }^{16}$ R. Natarajan and A. Acrivos, "The instability of the steady flow past spheres and disks," J. Fluid Mech. 254, 323 (1993).

${ }^{17} \mathrm{~B}$. Ghidersa and J. Dušek, "Breaking of axisymmetry and onset of unsteadiness in the wake of a sphere," J. Fluid Mech. 423, 33 (2000).

${ }^{18}$ B. Pier, "Local and global instabilities in the wake of a sphere," J. Fluid Mech. 603, 39 (2008)

${ }^{19}$ D. Fabre, F. Auguste, and J. Magnaudet, "Bifurcations and symmetry breaking in the wake of axisymmetric bodies," Phys. Fluids 20, 051702 (2008).

${ }^{20} \mathrm{C}$. Brücker, "Spatio-temporal reconstruction of vortex dynamics in axisymmetric wakes," J. Fluids Struct. 15, 543 (2001). 